\title{
Effects of Ti underlayer on the degree of order of $\mathrm{Fe}_{50} \mathrm{Pt}_{50}$ films
}

\author{
S.C. Chen ${ }^{\mathrm{a}, \mathrm{b}, *}$, P.C. Kuo ${ }^{\mathrm{a}}$, S.T. Kuo ${ }^{\mathrm{a}}$, A.C. Sun ${ }^{\mathrm{a}}$, C.T. Lie ${ }^{\mathrm{a}}$, C.Y. Chou ${ }^{\mathrm{a}}$ \\ a Institute of Materials Science and Engineering, National Taiwan University, Taipei, Taiwan \\ ${ }^{\mathrm{b}}$ Department of Mechanical Engineering, De Lin Institute of Technology, Taipei, Taiwan
}

Received 23 September 2002; received in revised form 6 January 2003; accepted 27 January 2003

\begin{abstract}
$\mathrm{Fe}_{50} \mathrm{Pt}_{50} / \mathrm{Ti}$ double-layer films were deposited on corning $1737 \mathrm{~F}$ glass substrates by d.c. magnetron sputtering of $\mathrm{FePt}$ and $\mathrm{Ti}$ targets. The magnetic layer FePt was deposited at substrate temperature of $600{ }^{\circ} \mathrm{C}$ in order to get ordered $\gamma_{1}$-FePt hard magnetic phase. It is found that the degree of order $(S)$ of FePt layer increases with increasing Ti underlayer thickness. The $S$ value for FePt single-layer film is about 0.68 , and it increases to about 0.76 as the thickness of the Ti underlayer increases to $150 \mathrm{~nm}$. The average grain size of the FePt film is largely reduced as the Ti underlayer is introduced. The average grain size of the single-layer FePt film is about $25 \mathrm{~nm}$, it will reduced to about $12 \mathrm{~nm}$ as $30 \mathrm{~nm}$ Ti underlayer is introduced, but the average grain size of FePt is increased as the thickness of Ti underlayer is increased. Magnetic measurement indicates that the in-plane coercivity $\left(\mathrm{Hc}_{\|}\right)$of the FePt film increases with increasing thickness of Ti underlayer, but the in-plane squareness $\left(S_{\|}\right)$decreases.
\end{abstract}

(C) 2003 Elsevier Science B.V. All rights reserved.

Keywords: Magnetron sputtering; $\mathrm{Fe}_{50} \mathrm{Pt}_{50} / \mathrm{Ti}$ double-layer films; Magnetic properties; Degree of order

\section{Introduction}

The FePt thin films with $\mathrm{L}_{0}$ ordered structure have received extensive attention due to their extremely high magnetic anisotropy constant $K_{\mathrm{u}}$. The $K_{\mathrm{u}}$ value of fully ordered FePt alloy can be as high as $7 \times 10^{7} \mathrm{erg} \mathrm{cm}^{-3}$ $[1,2]$. Generally, the crystal structure of FePt film which deposits at room temperature is face-centered cubic (f.c.c.) $\gamma$-FePt disordered phase and shows soft magnetic behavior $[3,4]$. This disordered f.c.c. $\gamma$-FePt phase can be transformed to hard magnetic face-centered-tetragonal (f.c.t.) $\gamma_{1}-\mathrm{FePt}$ ordered phase after annealing at high temperature [4,5]. Therefore, $\mathrm{Hc}$ value of the FePt film is dependent on the degree of order of the film, i.e., the content of ordered $\gamma_{1}-\mathrm{FePt}$ phase in the film. When the f.c.c. $\gamma$-FePt phase transforms to the $\gamma_{1}$-FePt phase, the lattice parameter is contractive along the $c$-axis, resulting in a $c / a$ ratio less than unity. The $c / a$ ratio is 0.956 for fully ordered $\gamma_{1}-\mathrm{FePt}$ phase [6]. The degree of order of the FePt film is increased as $c / a$ ratio is decreased.

\footnotetext{
* Corresponding author. Tel.: +886-2-2364-8881; fax: +886-22363-4562.

E-mail address: sscchh@ms28.hinet.net (S.C. Chen).
}

It has been shown that the introduction of Ti underlayer would improve the out-plane magnetic properties of the CoCrPt magnetic recording films $[7,8]$. In this work, we investigated the effects of Ti underlayer on the degree of order, microstructure and magnetic properties of the magnetic FePt layer, which was deposited on heated substrate in order to form ordered $\gamma_{1}$-FePt hard magnetic phase directly [9].

\section{Experimental}

The $\mathrm{Fe}_{50} \mathrm{Pt}_{50} / \mathrm{Ti}$ double-layer film was sputtered on corning $1737 \mathrm{~F}$ glass substrate by using high-purity FePt $(99.99 \%)$ and $\mathrm{Ti}(99.99 \%)$ targets with d.c. magnetron sputtering. The magnetic layer $\mathrm{FePt}$ was deposited at substrate temperature of $600{ }^{\circ} \mathrm{C}$ in order to get ordered $\gamma_{1}$-FePt hard magnetic phase. The thickness of $\mathrm{Ti}$ underlayer was varied from 30 to $150 \mathrm{~nm}$ and the magnetic layer was fixed at $300 \mathrm{~nm}$. Thickness of $\mathrm{Ti}$ underlayer was controlled by varying sputtering time. The substrate was rotated at $38 \mathrm{rpm}$ in order to attain a uniform composition of $\mathrm{FePt} / \mathrm{Ti}$ double films. A layer of $\mathrm{SiN}_{x}$ with $30 \mathrm{~nm}$ thickness was covered on the magnetic film to avoid the oxidation of magnetic layer. 
The base pressure of sputtering chamber was around $3 \times 10^{-7}$ Torr. The sputtering power for Ti underlayer was $120 \mathrm{~W}$ and the sputtering Ar pressure was fixed at 3 mTorr after introducing high-purity argon (99.999\%). The sputtering power for FePt magnetic layer was $40 \mathrm{~W}$ and Ar pressure was 10 mTorr.

Magnetic properties of the film were measured by using vibrating sample magnetometer (VSM) with maximum applied field of $12 \mathrm{kOe}$. Structure of the film was determined by X-ray diffractometer (XRD). The film microstructure was observed by field emission scanning electron microscopy (FE-SEM). Grain size of the film was derived from the XRD diffraction peak based on Scherrer equation. The composition and homogeneity of the magnetic film were analyzed by energy-dispersive spectrum (EDS). The film thickness was measured by atomic force microscope (AFM) and $\alpha$-step.

\section{Results and discussion}

Fig. 1 shows the XRD patterns of $\mathrm{Fe}_{50} \mathrm{Pt}_{50} / \mathrm{Ti}$ films with different Ti underlayer thickness. As thickness of the Ti underlayer increases from 0 to $50 \mathrm{~nm}$, intensity of the peak $\operatorname{Ti}\left(0_{0} 2\right)$ become stronger and the peak of Ti(l $\left.\begin{array}{lll}1 & 0\end{array}\right)$ appear. However, the $\left(\begin{array}{lll}0 & 0 & 1\end{array}\right)$ and (lll 110$)$ diffraction peaks of $\gamma_{1}-\mathrm{FePt}$ phase disappear and intensity of the $\gamma_{1}$-FePt $\left(\begin{array}{lll}1 & 1 & 1\end{array}\right)$ peak is raised. Intensity of the $\gamma_{1}$-FePt $\left(\begin{array}{lll}1 & 1 & 1\end{array}\right)$ peak increases as the Ti underlayer thickness is further increased from 50 to $150 \mathrm{~nm}$. This

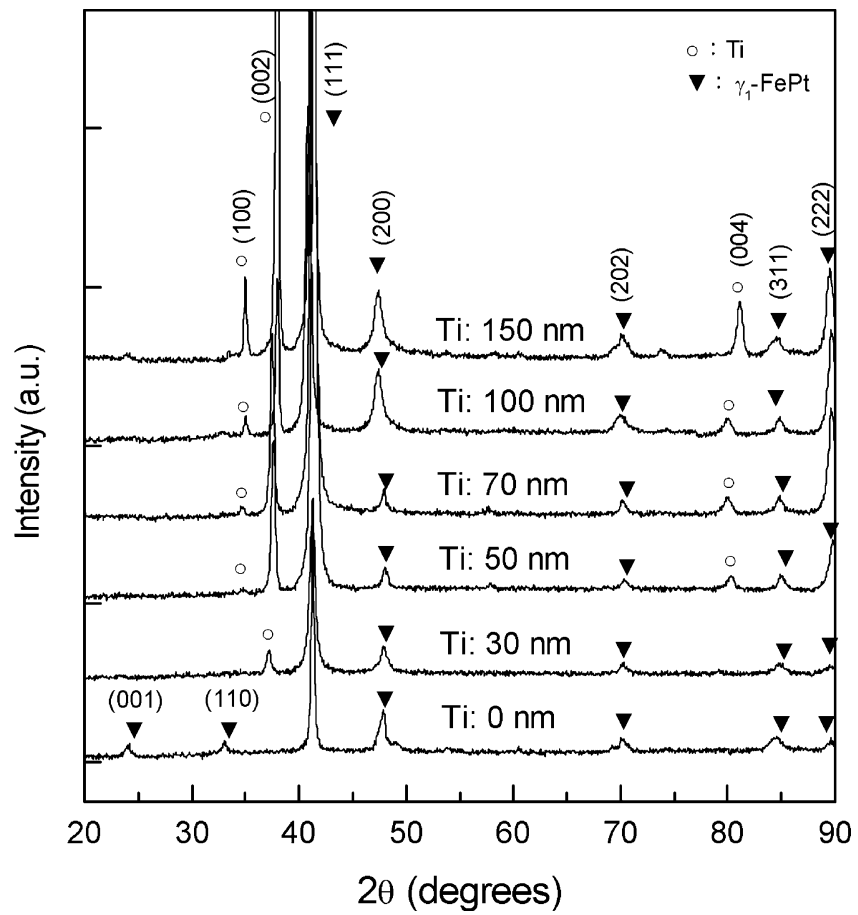

Fig. 1. The XRD patterns of various $\mathrm{Fe}_{50} \mathrm{Pt}_{50} / \mathrm{Ti}$ films with different Ti underlayer thickness. The thickness of FePt layer is fixed at $300 \mathrm{~nm}$. indicates that the amount of $\gamma_{1}-\mathrm{FePt}$ phase in the $\mathrm{FePt}$ layer increases and the preferred orientation of $\gamma_{1}-\mathrm{FePt}$ (1 111 ) is induced as the thickness of Ti underlayer is increased.

Fig. 2 shows the average grain sizes of FePt layer and Ti layer as a function of Ti underlayer thickness. The grain sizes of $\mathrm{FePt}$ and $\mathrm{Ti}$ are calculated from Scherrer equation [10] by using the diffraction peaks of $\gamma_{1}-\mathrm{FePt}$ (lll $\left.\begin{array}{lll}1 & 1\end{array}\right)$ and $\mathrm{Ti}\left(\begin{array}{lll}0 & 0 & 2\end{array}\right)$, respectively. We can see that the grain sizes of $\mathrm{FePt}$ and $\mathrm{Ti}$ are increased with increasing Ti underlayer thickness. Average grain size of the Ti underlayer increases from 18.2 to $27.1 \mathrm{~nm}$ and the average grain size of the FePt layer increases from 11.6 to $24 \mathrm{~nm}$ as thickness of Ti underlayer increases from 30 to $150 \mathrm{~nm}$. Average grain size of $\mathrm{FePt}$ without $\mathrm{Ti}$ underlayer is $24.3 \mathrm{~nm}$. However, the grain size of $\mathrm{FePt}$ decreases sharply from 24.3 to $11.6 \mathrm{~nm}$ as $30 \mathrm{~nm}$ of Ti underlayer is introduced. The decrease of FePt grain size when Ti underlayer is introduced is because the grain growth of FePt is along the grain of $\mathrm{Ti}$ underlayer. The grain size of FePt is controlled by the grain size of $\mathrm{Ti}$ underlayer. Since, the grain size of Ti increases with increasing Ti layer thickness as shown in Fig. 2, the grain size of FePt also increases with increasing Ti layer thickness.

Fig. 3 shows the FE-SEM micrograph of the crosssection of the $\mathrm{SiN}_{x} / \mathrm{FePt} / \mathrm{Ti}$ film with $\mathrm{Ti}$ underlayer thickness of $100 \mathrm{~nm}$. Since the sample is tilted slightly during preparation, the thickness of $\mathrm{SiN}_{x}$ layer looks like larger than $30 \mathrm{~nm}$. The Ti underlayer grows to columnar grain which is perpendicular to the film plane and the XRD analysis of Fig. 1 shows that the preferred orientation of Ti underlayer is $\left[\begin{array}{lll}0 & 0 & 2\end{array}\right]$. The grain growth of $\mathrm{FePt}$ is along the grain of $\mathrm{Ti}$ underlayer initially, columnar grains of $\mathrm{FePt}$ disappear and become ran-

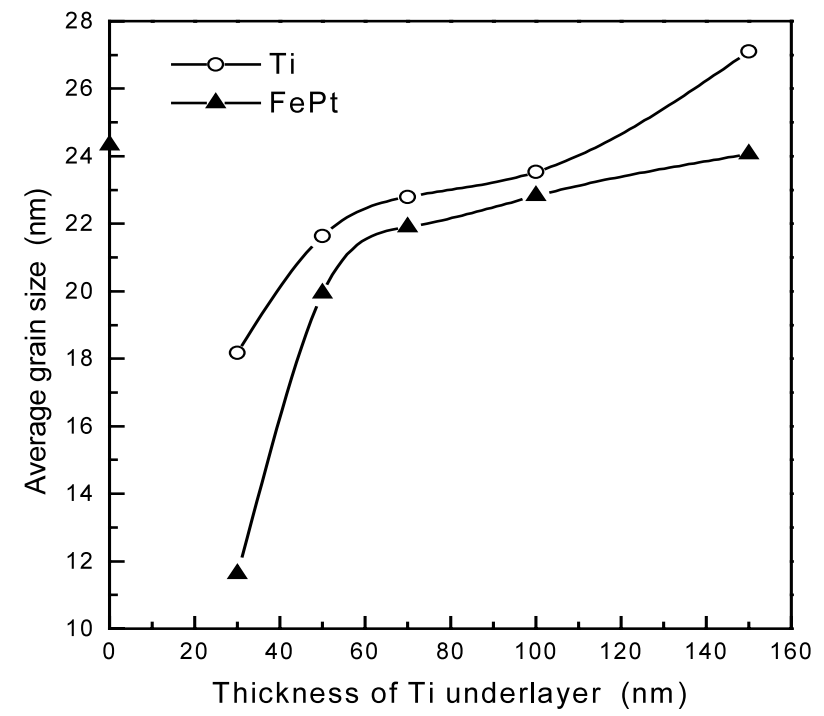

Fig. 2. The average grain sizes of FePt layer and Ti layer as a function of Ti underlayer thickness. 


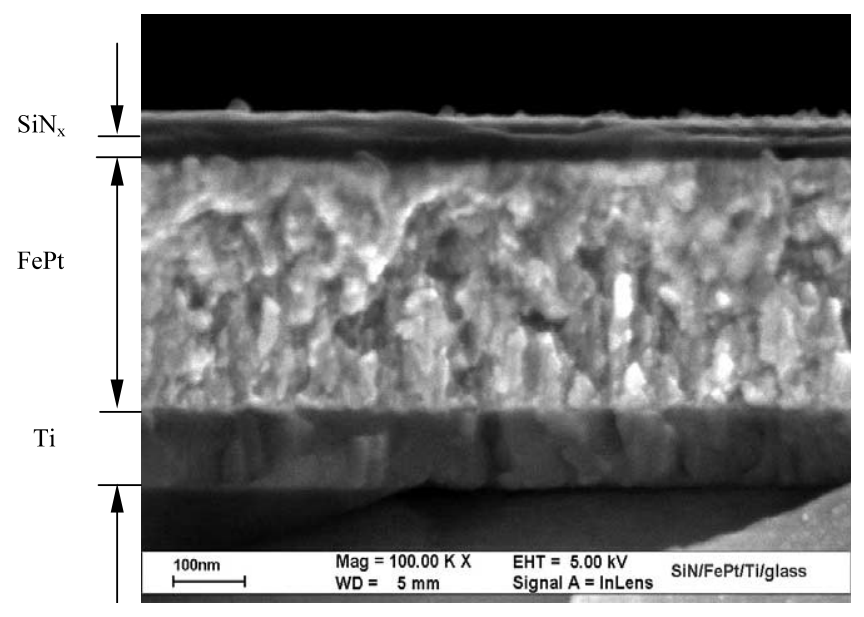

Fig. 3. FE-SEM micrograph of the cross-section of the $\mathrm{SiN}_{x} / \mathrm{FePt} / \mathrm{Ti}$ film. Thickness of $\mathrm{SiN}_{x}, \mathrm{FePt}$ and Ti layers are 30, 300 and $100 \mathrm{~nm}$, respectively.

domly orientated when FePt layer is thicker than about $150 \mathrm{~nm}$ as shown in Fig. 3.

The degree of order $(S)$ of the FePt magnetic layer will affect the magnetic properties of $\mathrm{FePt} / \mathrm{Ti}$ doublelayer films. The $c / a$ values of partially ordered $\mathrm{FePt}$ films with different thickness of $\mathrm{Ti}$ underlayer were determined by using the X-ray diffraction peaks of (1 111 ) and (2 00 ) of $\gamma_{1}$-FePt phase. Degree of order of the FePt layer can be obtained and expressed as [11]:

$$
S^{2}=\frac{1-c / a}{1-(c / a)_{S_{\mathrm{f}}}}
$$

where $(c / a)_{S_{\mathrm{f}}}$ is the $c / a$ value of fully ordered FePt film, it is 0.956 [6]. $(c / a)$ is the value of partially ordered $\mathrm{FePt}$ film. Fig. 4 shows the degree of order as a function of $\mathrm{Ti}$ underlayer thickness. The result shows the degree of order of FePt film increases with increasing the thick-

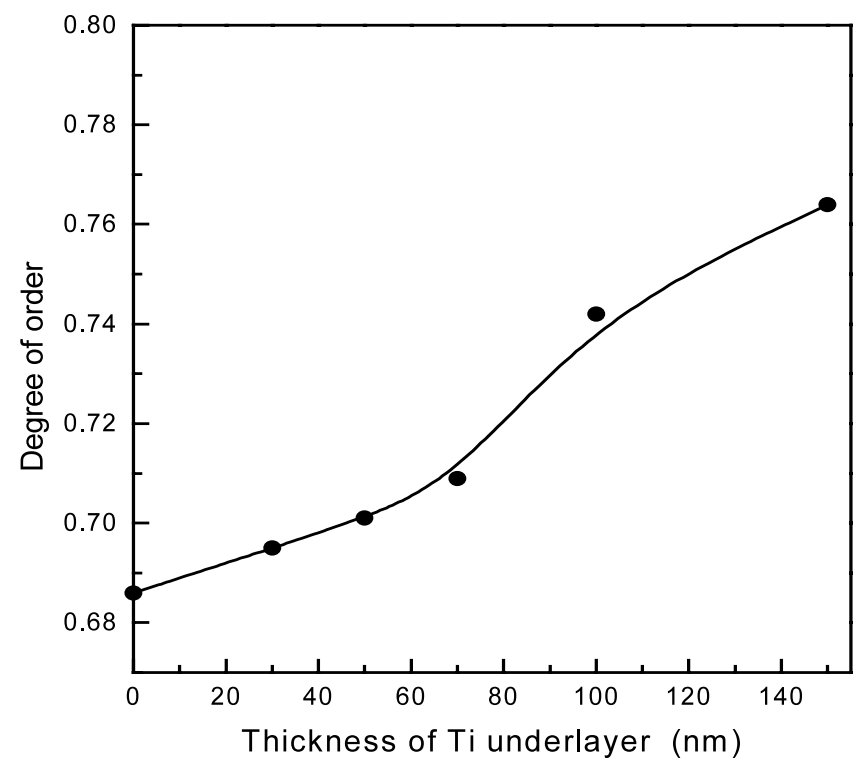

Fig. 4. The degree of order of FePt film as a function of the thickness of Ti underlayer in FePt/Ti film. ness of Ti underlayer. This means that the introduction of $\mathrm{Ti}$ underlayer and an increase in $\mathrm{Ti}$ underlayer thickness will increase the degree of order of the FePt film. The degree of order of $\mathrm{FePt}$ film without $\mathrm{Ti}$ underlayer is 0.684 . However, it increases to 0.741 as the thickness of Ti underlayer is $100 \mathrm{~nm}$, as shown in Fig. 4. When the thickness of Ti underlayer is further increased from 100 to $150 \mathrm{~nm}$, the degree of order of FePt film increases from 0.741 to 0.762 . The increase in the degree of order of FePt film is due to the grain sizes of $\mathrm{Ti}$ underlayer and FePt layer increase with increasing the thickness of Ti underlayer, as shown in Fig. 2. Wong et al. [12] had shown that the degree of interfacial misfit is larger as the grain size is larger in $\mathrm{Co} / \mathrm{Cr}$ film, and this will introduce stacking fault and misfit dislocation into the Co layer in order to reduce the degree of interfacial misfit, and the phase transformation is easier owing to the existence of these defects. Similarly, in our FePt/Ti system, the introduction of these defects in the $\mathrm{FePt}$ layer will make the transformation of $\gamma$-FePt into $\gamma_{1^{-}}$ $\mathrm{FePt}$ phase easier. Therefore, the degree of order of $\mathrm{FePt}$ layer is increased with increasing Ti underlayer thickness, i.e., the grain size of $\mathrm{Ti}$, as shown in Fig. 4.

Fig. 5 shows the in-plane coercivity $\left(\mathrm{Hc}_{\|}\right)$and perpendicular coercivity $\left(\mathrm{Hc}_{\perp}\right)$ as function of the $\mathrm{Ti}$ underlayer thickness. It indicates that $\mathrm{Hc}_{\|}$and $\mathrm{Hc}_{\perp}$ of the film increase with increasing thickness of $\mathrm{Ti}$ underlayer. For the FePt single-layer film without Ti underlayer, $\mathrm{Hc}_{\|}$and $\mathrm{Hc}_{\perp}$ are 3.1 and $2.3 \mathrm{kOe}$, respectively. As the thickness of Ti underlayer increases to $100 \mathrm{~nm}, \mathrm{Hc}_{\|}$ and $\mathrm{Hc}_{\perp}$ will increase to 7.3 and $4.2 \mathrm{kOe}$, respectively. Since increasing the thickness of $\mathrm{Ti}$ underlayer will enhance the degree of order of FePt layer, i.e., increase the amount of $\gamma_{1}$-FePt phase in the FePt layer, the in-

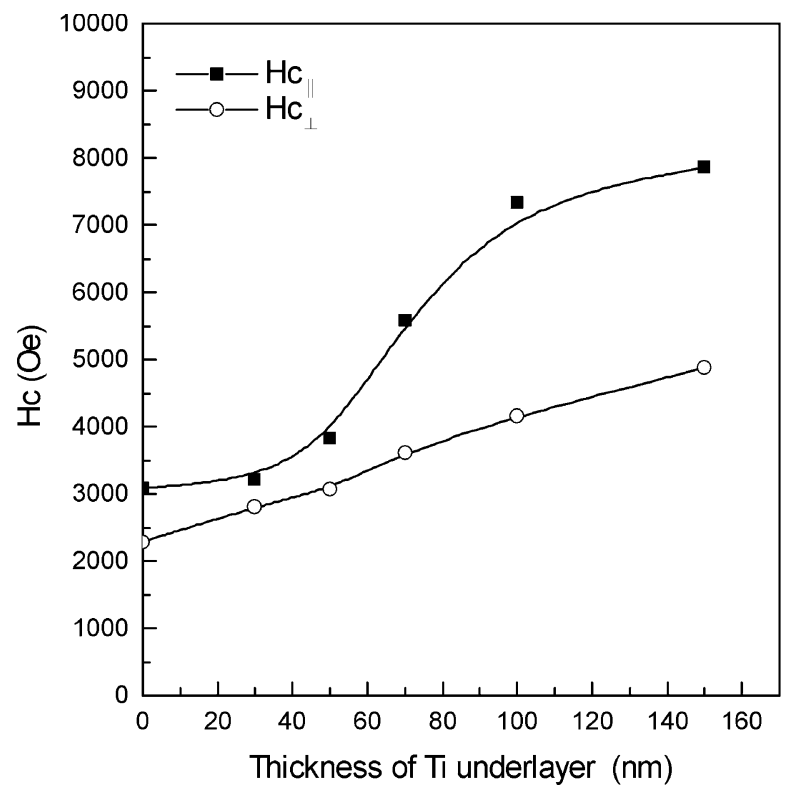

Fig. 5. $\mathrm{Hc}_{\|}$and $\mathrm{Hc}_{\perp}$ of the $\mathrm{FePt} / \mathrm{Ti}$ film as a function of Ti underlayer thickness. 


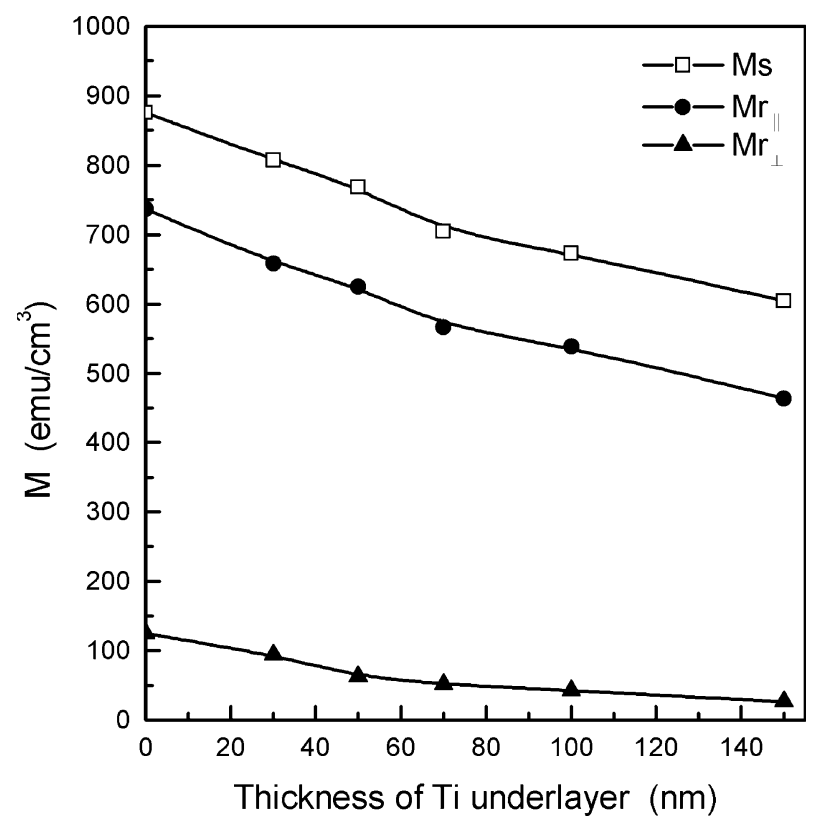

Fig. 6. Ms, $\mathrm{Mr}_{\|}$and $\mathrm{Mr}_{\perp}$ of the FePt/Ti film as a function of the thickness of $\mathrm{Ti}$ underlayer.

plane and perpendicular coercivity of the film are increased.

Fig. 6 shows the saturation magnetization (Ms), inplane remnant magnetization $\left(\mathrm{Mr}_{\|}\right)$and perpendicular remnant magnetization $\left(\mathrm{Mr}_{\perp}\right)$ of the $\mathrm{FePt} / \mathrm{Ti}$ films as a function of the $\mathrm{Ti}$ underlayer thickness. It shows that Ms, $\mathrm{Mr}_{\|}$and $\mathrm{Mr}_{\perp}$ of the film decrease with increasing thickness of $\mathrm{Ti}$ underlayer. For FePt single-layer film without Ti underlayer, the $\mathrm{Ms}, \mathrm{Mr}_{\|}$and $\mathrm{Mr}_{\perp}$ values are 875,736 and $124 \mathrm{emu} \mathrm{cm}{ }^{-3}$, respectively. $\mathrm{Ms}, \mathrm{Mr}_{\|}$and $\mathrm{Mr}_{\perp}$ values will decrease to 672,538 and $50 \mathrm{emu} \mathrm{cm}^{-3}$, respectively, as $100 \mathrm{~nm}$ Ti underlayer is introduced. The

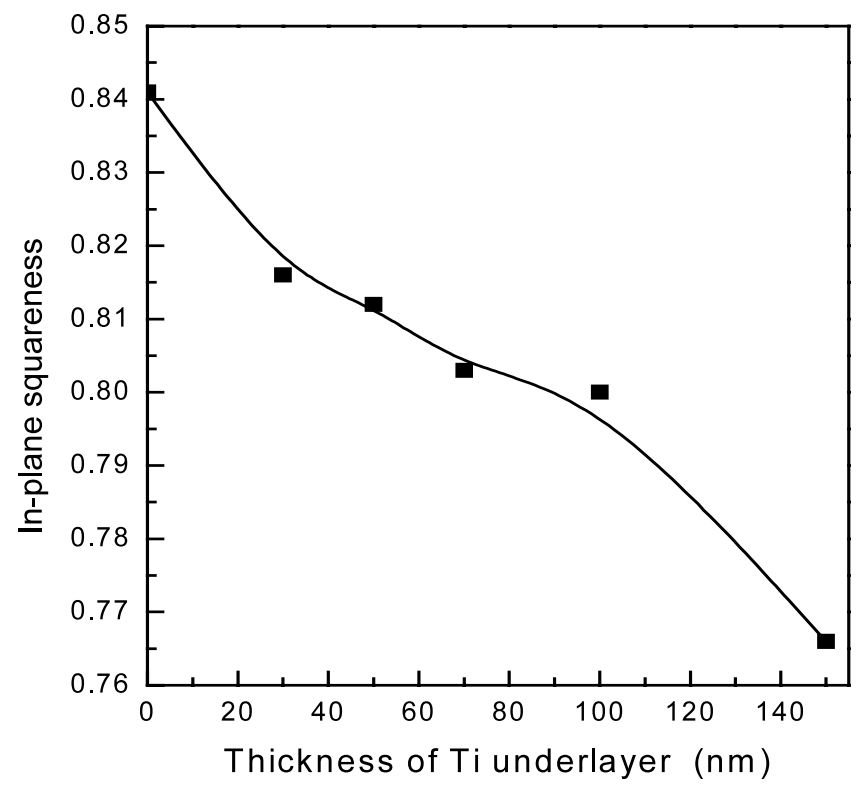

Fig. 7. The relationship between in-plane squareness and the thickness of Ti underlayer of $\mathrm{FePt} / \mathrm{Ti}$ film. decrease in magnetization of FePt layer with increasing Ti underlayer thickness is mainly due to the increase of $\gamma_{1}$-FePt phase content in the film, because the Ms value of $\gamma_{1}$-FePt phase is lower than that of $\gamma$-FePt phase [13].

Fig. 7 shows the relationship between in-plane squareness $\left(S_{\|}\right)$and the thickness of $\mathrm{Ti}$ underlayer of the FePt/Ti film. $S_{\|}$is defined as the ratio of $\mathrm{Mr}_{\|}$to Ms. It shows that $S_{\|}$decreases with increasing Ti underlayer thickness. $S_{\|}$value is 0.84 for the FePt single-layer film without Ti underlayer. As the thickness of Ti underlayer increases from 0 to $100 \mathrm{~nm}, S_{\|}$decreases from 0.84 to 0.8. Obviously, thicker Ti underlayer has not favored the in-plane squareness of FePt/Ti film.

\section{Conclusions}

The conclusions are as follows: (1) The degree of order and $\mathrm{Hc}_{\|}$of the FePt/Ti double-layer films, both increase with increasing thickness of Ti underlayer, and (2) although the increase of $\mathrm{Ti}$ underlayer thickness enhances the degree of order of the FePt layer in FePt/Ti film, the in-plane squareness and saturation magnetization decrease with increasing thickness of Ti underlayer.

\section{Acknowledgements}

This work was supported by the National Science Council of ROC through Grant No. NSC90-2216-E002-036.

\section{References}

[1] K. Watanabe, H. Masumoto, Trans. Jpn. Inst. Met. 24 (1983) 627.

[2] M. Watanabe, T. Nakayama, K. Watanabe, T. Hirayama, A. Tonomura, Mater. Trans. JIM 37 (1996) 489.

[3] J.A. Aboaf, T.R. McGuire, S.R. Herd, E. Klokholm, IEEE Trans. Magn. 20 (1984) 1642.

[4] S.C. Chen, P.C. Kuo, A.C. Sun, C.T. Lie, W.C. Hsu, Mater. Sci. Eng. B 88 (2002) 91.

[5] K. Watanabe, Mater. Trans. JIM 32 (1991) 292.

[6] P. Villas, L.D. Calvert, Pearson's Handbook of Crystallographic Data for Intermetallic Phase, vol. 4, ASM Information, 1991.

[7] I.S. Lee, H. Ryu, H.J. Lee, T.D. Lee, J. Appl. Phys. 85 (1999) 6133.

[8] B. Lu, T. Klemmer, S. Khizroev, J.K. Howard, D. Litvinov, A.G. Roy, D.E. Laughlin, IEEE Trans. Magn. 37 (2001) 1319.

[9] S. Jeong, T. Ohkubo, A.G. Roy, D.E. Laughlin, M.E. McHenry, J. Appl. Phys. 91 (2002) 6863.

[10] B.D. Cullity, Elements of X-ray Diffraction, 2nd ed., AddisonWesley, Reading, MA, 1978.

[11] B.W. Robarts, Acta Metall. 2 (1954) 597.

[12] B.Y. Wong, J.F. Ying, K. Johnson, IEEE Trans. Magn. 36 (2000) 2360.

[13] T. Katayama, T. Sugimoto, Y. Suzuki, M. Hashimoto, P. dc Haan, J.C. Lodder, J. Magn. Magn. Mater. 104-107 (1992) 1002 . 